\title{
Coincidence and common fixed point results for $\beta$-quasi contractive mappings on metric spaces endowed with binary relation
}

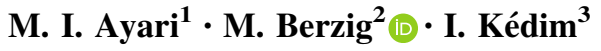

Received: 7 March 2016/Accepted: 3 June 2016/Published online: 1 July 2016

(c) The Author(s) 2016. This article is published with open access at Springerlink.com

\begin{abstract}
Coincidence and common fixed point theorems for $\beta$-quasi contractive mappings on metric spaces endowed with binary relations and involving suitable comparison functions are presented. Our results generalize, improve, and extend several recent results. As an application, we study the existence of solutions for some class of integral equations.
\end{abstract}

Keywords Fixed point theory - Transitive binary relation . Ordered metric spaces

Mathematics Subject Classification $47 \mathrm{H} 10 \cdot 54 \mathrm{H} 25$

\section{Introduction}

The Banach contraction principle [1] may be considered as one of most powerful tools for establishing existence and uniqueness of solutions for various non linear problems.

M. Berzig

maher.berzig@ept.rnu.tn; maher.berzig@gmail.com

M. I. Ayari

iadh_ayari@yahoo.com

I. Kédim

imed.kedim@gmail.com

1 Carthage University, Institu National Des Sciences Appliquée et de Technologie de Tunis, Centre Urbain Nord, BP 676-1080 Tunis, Tunisia

2 Tunis University, École Nationale Supérieure d'Ingénieurs de Tunis, 5 Avenue Taha Hussein, BP 56, Bab Manara, 1008 Tunis, Tunisia

3 Carthage University, Faculté des Sciences de Bizerte, 7021 Bizerte, Zarzouna, Tunisia
This principle is often used in the analysis of nonlinear governing equations arising in physics, engineering, economy and other disciplines. In its statement that every mapping $T: X \rightarrow X$ has a unique fixed point in the setting of nonempty complete metric spaces $(X, d)$, the principle is restricted to mappings that satisfy a contraction given by $d(T x, T y) \leq q d(x, y)$ where $q \in(0,1)$ and $x, y \in X$. In order to generalize the Banach contraction principle, Ćirić in [2] shows that $T$ still admits a unique fixed point if $T$ satisfies a quasi contractive condition of the form:

$d(T x, T y) \leq q \max \{d(x, y), d(x, T x), d(y, T y), d(x, T y), d(y, T x)\}$,

for all $x, y \in X$. It is worthwhile noting that the quasi-contractive condition subsumes the original contraction and may also be applied to non continuous mappings. Later, Samet and Turinici [3], established an important fixed point result where the contractive condition is required only on binary related elements of $X$. Several known results from literature can be derived from their main theorem, for instance we cite those of Kannan [4], Chatterjea [5], Hardy and Rogers [6], Ran and Reurings [7], Nieto and López [8], Ćirić [9] and Kirk et al. [10]. More recently, there have been some attempts to generalize the fixed point results for metric spaces endowed with binary relation. For more details, we refer the reader to [11-19].

In this paper, some coincidence point results in complete metric spaces endowed with transitive binary relations are first highlighted. Subsequently, the existence and uniqueness of common fixed point theorem for two mappings are established under some suitable conditions. Moreover, some coincidence and common fixed point theorems involving amorphous binary relation are proven. Finally, as an application, existence study of solutions to some integral 
equation is presented. The paper is divided into five sections. Section introduces the notation used herein, presents a number of definitions and recalls some useful results. Coincidence and common fixed point theorems are stated in Sect. 1, while their proofs are the subject of Sect. 2. Several consequences are subsequently derived in Sect. 3. Finally, the existence of solutions for some class of Urysohn integral equation is shown in Sect. 4.

\section{Preliminaries}

Let us introduce some definitions and recall some basic preliminary results which will be needed in the following sections. Throughout this paper, we denote by $\mathbb{N}$ the set of all positive integer and $\mathbb{N}_{0}=\mathbb{N} \cup\{0\}$. Let $(X, d)$ be a metric space, $\mathcal{R}$ be a binary relation on $X$ and $g, T: X \rightarrow X$ be two mappings. Denote by

$C(g, T):=\left\{x_{*} \in X: g x_{*}=T x_{*}\right\}$.

the set of coincidence points of $g$ and $T$. Let $x_{0} \in X$, and suppose that $T(X) \subseteq g(X)$. Hence, we can choose $x_{1} \in X$ satisfying $g x_{1}=T x_{0}$. Again from $T(X) \subseteq g(X)$, we can choose $x_{2} \in X$ satisfying $g x_{2}=T x_{1}$. Continuing this process, we construct a sequence $\left\{x_{n}\right\}$ satisfying

$g x_{n+1}=T x_{n} \quad$ for all $n \in \mathbb{N}_{0}$.

Denote by $\mathcal{O}\left(x_{0}\right)$ the set of all sequences $\left\{x_{n}\right\}$ satisfying (2). Define also the $g$-orbit set, at $s=\left\{x_{k}\right\}_{k \in \mathbb{N}_{0}} \in \mathcal{O}\left(x_{0}\right)$, from $p$ to $q$ with $q>p>0$, by $O_{p, q}(g, s)=\left\{g x_{p}, g x_{p+1}\right.$, $\left.\ldots, g x_{q}\right\}$. Similarly, define the infinite $g$-orbit set at $s$ from $p$ by $O_{p, \infty}(g, s)=\left\{g x_{p}, g x_{p+1}, g x_{p+2}, \ldots\right\}$.

Definition 1.1 We say that $(X, d)$ is $g$-orbitally complete if and only if for all $x_{0} \in X$ and $s \in \mathcal{O}\left(x_{0}\right)$. every Cauchy sequence in $O_{0, \infty}(g, s)$ converges in $X$.

Definition 1.2 ([11]) A subset $D$ of $X$ is called $\mathcal{R}$ - $g$-directed if for every $x, y \in D$, there exists $z \in X$ such that gx $\operatorname{R} g z$ and $g y \mathcal{R} g z$.

Definition 1.3 ([3]) We say that $(X, d, \mathcal{R})$ is regular if for a sequence $\left\{x_{n}\right\}$ in $X$, if we have $x_{n} \mathcal{R} x_{n+1}$ for all $n \in \mathbb{N}_{0}$ and $\lim _{n \rightarrow \infty} d\left(x_{n}, x\right)=0$ for some $x \in X$, then there exists a subsequence $\left\{x_{n(k)}\right\}$ of $\left\{x_{n}\right\}$ such that $x_{n(k)} \mathcal{R} x$ for all $k \in \mathbb{N}_{0}$.

Definition 1.4 ([11]) Let $X$ be a non empty set, $g, T$ : $X \rightarrow X$ be two mappings and $\mathcal{R}$ be a binary relation. We say that $T$ is $g$-comparative if

$x, y \in X, \quad g x \mathcal{R} g y \Longrightarrow T x \mathcal{R} T y$.

Definition 1.5 Let $\beta \in(0,+\infty)$ and $\varphi:[0,+\infty) \rightarrow$ $[0,+\infty)$ be a function satisfying the properties:

(P1) $\varphi$ is nondecreasing;
(P2) $\lim _{n \rightarrow \infty} \varphi_{\beta}^{n}(t)=0$ for all $t>0$, where $\varphi_{\beta}^{n}$ denote the $n$-th iterate of $\varphi_{\beta}$ and $\varphi_{\beta}(t)=\varphi(\beta t)$;

(P3) there exists $s \in(0,+\infty)$ such that $\sum_{n=1}^{\infty} \varphi_{\beta}^{n}(s)$ $<\infty$;

(P4) $\quad\left(i d-\varphi_{\beta}\right) \circ \varphi_{\beta}(t) \leq \varphi_{\beta} \circ\left(i d-\varphi_{\beta}\right)(t)$ forallt $\geq 0$, where id: $[0,+\infty) \rightarrow[0,+\infty)$ is the identity function.

The set of all functions $\varphi$ satisfying (P1)-(P4) is called the set of $\beta$-comparison functions and denoted by $\Phi_{\beta}$.

Example 1.1 Let $\varphi:[0,+\infty) \rightarrow[0,+\infty)$ be a function given by $\varphi(t)=a t$ where $a \in\left(0, \beta^{-1}\right)$ and $\beta>0$, then $\varphi \in$ $\Phi_{\beta}$. Note that, if $a>1$ then $\varphi(t)>t$ and so $\sum_{n=1}^{\infty} \varphi^{n}(t)=\infty$.

Lemma 1.1 Let $\beta \in(0,+\infty)$ and $\varphi \in \Phi_{\beta}$. Then

(i) $\varphi_{\beta}$ is nondecreasing;

(ii) $\varphi_{\beta}(t)<t$ for all $t>0$;

(iii) $\sum_{n=1}^{\infty} \varphi_{\beta}^{n}(t)<\infty$ for all $t>0$;

(iv) $\quad\left(\right.$ id $\left.-\varphi_{\beta}\right) \circ \varphi_{\beta}^{n}(t) \leq \varphi_{\beta}^{n} \circ\left(\right.$ id $\left.-\varphi_{\beta}\right)(t)$ for all $t \geq 0$ and $n \in \mathbb{N}_{0}$.

Proof From $t<t^{\prime}$, it follows that $\beta t<\beta t^{\prime}$. Using (P1), we obtain $\varphi(\beta t)<\varphi\left(\beta t^{\prime}\right)$, that is $\varphi_{\beta}(t)<\varphi_{\beta}\left(t^{\prime}\right)$ which proves (i). Suppose next that (ii) does not hold. There exists $t_{0}>0$ such that $\varphi_{\beta}\left(t_{0}\right) \geq t_{0}$ and $\beta \varphi_{\beta}\left(t_{0}\right) \geq \beta t_{0}$. Using (i), it follows $\varphi_{\beta}^{2}\left(t_{0}\right) \geq \varphi_{\beta}\left(t_{0}\right) \geq t_{0}$. Therefore, by induction, we obtain $\varphi_{\beta}^{n}\left(t_{0}\right) \geq t_{0}$ which contradict (P2), and hence (ii) is satisfied. Next, from (P3), there exists $s \in(0,+\infty)$ such that $\sum_{n=1}^{\infty} \varphi_{\beta}^{n}(s)<\infty$. Since (P2) holds, for $t>0$ there exists $\quad \ell \in \mathbb{N}$ such that $\varphi_{\beta}^{\ell}(t) \leq \varphi_{\beta}(s)$. Hence, $\varphi_{\beta}^{\ell+n}(t) \leq \varphi_{\beta}^{n}(s)$ for all $n \in \mathbb{N}_{0}$. Which means that,

$\sum_{n=1}^{\infty} \varphi_{\beta}^{n}(t)=\sum_{n=1}^{\ell} \varphi_{\beta}^{n}(t)+\sum_{n=1}^{\infty} \varphi_{\beta}^{\ell+n}(t) \leq \sum_{n=1}^{\ell} \varphi_{\beta}^{n}(t)+\sum_{n=1}^{\infty} \varphi_{\beta}^{n}(s)<\infty$,

and this proves (iii). Finally, (iv) follows from (P4) using an induction on $n$.

Remark 1.1 Let $\alpha, \beta \in(0,+\infty)$. If $\alpha<\beta$, then $\Phi_{\beta} \subset \Phi_{\alpha}$.

Definition 1.6 Let $X$ be a non empty set and $g: X \rightarrow X$ be a mapping. A mapping $T: X \rightarrow X$ is called $\beta$-quasi contractive (with respect to $g$ ) if there exist $\beta>0$ and $\varphi \in \Phi_{\beta}$ such that $x, y \in X: \operatorname{gx} \mathcal{R} g y \Longrightarrow d(T x, T y) \leq \varphi\left(M_{T, g}(x, y)\right)$,

where

$$
\begin{gathered}
M_{T, g}(x, y)=\max \left\{\alpha_{0} d(g x, g y), \alpha_{1} d(g x, T x), \alpha_{2} d(g y, T y),\right. \\
\left.\alpha_{3} d(g x, T y), \alpha_{4} d(g y, T x)\right\},
\end{gathered}
$$

with $\alpha_{k} \geq 0$ for $0 \leq k \leq 4$. 


\section{Main results}

The basic definitions about coincidence points, $g$-orbital completeness of metric binary spaces, $g$-comparative mappings, directed subsets and regular spaces endowed with relations, were stated in the previous section. In addition, we introduced concepts of $\beta$-comparison functions and $\beta$-quasi contractive with respect to $g$. In the current section, we state the main results of coincidence points for mappings in complete metric spaces endowed with binary relations. The proofs supporting these results will be the subject of the next section. We start with the first result on the existence of coincidence points.

Theorem 2.1 Let $(X, d)$ be a g-orbitally complete space endowed with a transitive binary relation $\mathcal{R}$ such that $(X, d, \mathcal{R})$ is regular. Let $g, T: X \rightarrow X$ be two mappings satisfying the following conditions:

(A1) there exists $x_{0} \in X$ such that $g x_{0} \mathcal{R} T x_{0}$;

(A2) $g(X)$ is closed and $T(X) \subset g(X)$;

(A3) $T$ is $g$-comparative;

(A4) there exists $\beta \geq \max _{0 \leq k \leq 4}\left\{\alpha_{k}\right\}$ such that $T$ is $\beta$ quasi contractive (w.r.t. g);

Moreover, assume that one of the following conditions holds:

(A5.a) $\quad \varphi$ is continuous;

(A5.b) $\quad \beta>\max \left\{\alpha_{2}, \alpha_{3}\right\}$;

(A5.c) Tand $g$ are continuous and commute on $O_{\infty}(g, s)$ for some $s \in \mathcal{O}\left(x_{0}\right)$.

Then, $T$ and $g$ have a coincidence point in $X$.

In the following theorem, some supplementary conditions to those of Theorem 2.1 have been added, in order to show existence and uniqueness of common fixed point.

Theorem 2.2 In addition to the hypotheses of Theorem 2.1, assume that the following conditions hold:

(B1) $\mathcal{R}$ is symmetric or $\beta \geq \max _{0 \leq k \leq 4}\left\{\alpha_{k}, 2 \alpha_{1}\right\}$;

(B2) $\quad C(g, T)$ is $\mathcal{R}$-g-directed;

(B3) $T$ and $g$ commute at their coincidence points.

Then, $T$ and $g$ have a unique common fixed point.

Next, we use an amorphous binary relation instead of transitive binary relation. However, the results are obtained in the framework of complete metric spaces.

Theorem 2.3 Let $(X, d)$ be a complete metric space endowed with a binary relation $\mathcal{R}$ such that $(X, d, \mathcal{R})$ is regular. Let $g, T: X \rightarrow X$ be two mappings satisfying the following conditions:

(C1) There exists $x_{0} \in X$ such that $g x_{0} \mathcal{R} T x_{0}$;

(C2) $\quad g(X)$ is closed and $T(X) \subset g(X)$;
(C3) $T$ is g-comparative;

(C4) There exists $\beta \geq \max _{0 \leq k \leq 4}\left\{\alpha_{k}, 2 \alpha_{4}\right\}$ such $T$ is $\beta$ quasi contractive (w.r.t. g);

Moreover, assume that one of the following conditions holds:

(C5.a) $\varphi$ is continuous;

(C5.b) $\quad \beta>\max \left\{\alpha_{2}, \alpha_{3}\right\}$.

Then $T$ and $g$ have a coincidence point in $X$.

Proceeding as before, the existence and uniqueness of a common fixed points can be shown under some supplementary conditions, as stated in the following theorem.

Theorem 2.4 In addition to the hypotheses of Theorem 2.3, suppose that,

(D1) $\quad C(g, T)$ is $\mathcal{R}$-g-directed;

(D2) $T$ and $g$ commute at their coincidence points;

(D3) $\beta \geq \max \left\{\alpha_{0}, 2 \alpha_{1}, 2 \alpha_{3}, 2 \alpha_{4}\right\}$.

Then, $T$ and $g$ have a unique common fixed point.

\section{Proofs}

The proof of the main results are postponed after several preliminary lemmas.

Lemma 3.1 Let $(X, d)$ be a metric space endowed with binary relation $\mathcal{R}$ and $g, T: X \rightarrow X$ be two mappings satisfying:

(a1) there exists $x_{0} \in X$ such that $g x_{0} \mathcal{R} T x_{0}$;

(a2) $T(X) \subset g(X)$;

(a3) $T$ is g-comparative;

(a4) $T$ is $\beta$-quasi contractive (w.r.t. g).

Then, for any $s=\left\{x_{n}\right\}_{n \in \mathbb{N}} \in \mathcal{O}\left(x_{0}\right)$, we have

$d\left(g x_{i}, g x_{i+1}\right) \leq \varphi\left(M_{T, g}\left(x_{i-1}, x_{i}\right)\right), \quad$ for all $\mathrm{i} \in \mathbb{N}$.

Furthermore, if $\mathcal{R}$ is transitive then for all $i, j \in \mathbb{N}$, we have

$d\left(g x_{i}, g x_{j}\right) \leq \varphi\left(M_{T, g}\left(x_{i-1}, x_{j-1}\right)\right)$.

Proof It will suffice to show that

$g x_{i-1} \mathcal{R} g x_{i}, \quad$ for all $\mathrm{i} \in \mathbb{N}$,

where the sequence $\left\{x_{n}\right\}$ is given by (2). We proceed by induction on $i$. For $i=1$, we have from (a1), $g x_{0} \mathcal{R} T x_{0}$ and since $g x_{1}=T x_{0}$, then $g x_{0} \mathcal{R} g x_{1}$. We assume then that (4) holds for $i=k$, that is, $g x_{k-1} \mathcal{R} g x_{k}$ and prove that (4) is true for $i=k+1$. Since $T$ is $g$-comparative, then from $g x_{k-1} \mathcal{R} g x_{k}$ it follows $T x_{k-1} \mathcal{R} T x_{k}$. Therefore, by (2), we obtain $g x_{k} \mathcal{R} g x_{k+1}$. 
Lemma 3.2 In addition to the hypotheses of Lemma 3.1, suppose that $\beta \geq \max _{k=0 . .4}\left\{\alpha_{k}\right\}$ and $\mathcal{R}$ is transitive. Then, for $s=\left\{x_{n}\right\}_{n \in \mathbb{N}_{0}} \in \mathcal{O}\left(x_{0}\right)$, we have

$d\left(g x_{i}, g x_{j}\right) \leq \varphi_{\beta}\left(D\left(O_{0, n}(g, s)\right)\right), \quad$ for all $\mathrm{i}, \mathrm{j} \in\{1, \ldots, \mathrm{n}\}$,

where $D\left(O_{0, n}(g, s)\right)=\max \left\{d\left(g x_{i}, g x_{j}\right): 0 \leq i, j \leq n\right\}$.

Further, there exists $1 \leq k \leq n$ such that $D\left(O_{0, n}(g, s)\right)=$ $d\left(g x_{0}, g x_{k}\right)$.

Proof Let $1 \leq i<j \leq n$, using Definition 1.5 and Lemma 3.1 , we have

$$
\begin{aligned}
d\left(g x_{i}, g x_{j}\right)=d\left(T x_{i-1}, T x_{j-1}\right) & \leq \varphi\left(M_{T, g}\left(x_{i-1}, x_{j-1}\right)\right) \\
& \leq \varphi\left(\beta D\left(O_{0, n}(g, s)\right)\right) \\
& =\varphi_{\beta}\left(D\left(O_{0, n}(g, s)\right)\right) .
\end{aligned}
$$

In particular, by Lemma 1.1 , we have

$$
d\left(g x_{i}, g x_{j}\right) \leq \varphi_{\beta}\left(D\left(O_{0, n}(g, s)\right)\right)<D\left(O_{0, n}(g, s)\right)
$$

for all $1 \leq \mathrm{i}<\mathrm{j} \leq \mathrm{n}$,

which implies that $D\left(O_{0, n}(g, s)\right)=d\left(g x_{0}, g x_{k}\right)$ for some integer $k \leq n$.

Lemma 3.3 Under the same hypotheses of Lemma 3.2, we have

$D\left(O_{0, \infty}(g, s)\right) \leq \sum_{\ell=0}^{\infty} \varphi_{\beta}^{\ell}\left(d\left(g x_{0}, T x_{0}\right)\right)$,

where $D\left(O_{0, \infty}(g, s)\right):=\sup \left\{d\left(g x_{i}, g x_{j}\right), i, j \geq 0\right\}$.

Proof At first, we note that we have

$D\left(O_{0,1}(g, s)\right) \leq D\left(O_{0,2}(g, s)\right) \leq \cdots \leq D\left(O_{0, k}(g, s)\right)$

$\leq \cdots$ for all $k \in \mathbb{N}$.

Now, by Lemma 3.2 and the triangular inequality, we may write

$$
\begin{aligned}
D\left(O_{0, n}(g, s)\right)=d\left(g x_{0}, g x_{k}\right) & \leq d\left(g x_{0}, g x_{1}\right)+d\left(g x_{1}, g x_{k}\right) \\
& \leq d\left(g x_{0}, T x_{0}\right)+d\left(g x_{1}, g x_{k}\right) \\
& \leq d\left(g x_{0}, T x_{0}\right)+\varphi_{\beta}\left(D\left(O_{0, n}(g, s)\right)\right)
\end{aligned}
$$

and therefore

$\left(i d-\varphi_{\beta}\right)\left(D\left(O_{0, n}(g, s)\right)\right) \leq d\left(g x_{0}, T x_{0}\right)$.

Thus, by applying the nondecrasing map $t \mapsto \sum_{\ell=0}^{p} \varphi_{\beta}^{\ell}(t)$ to both side of the previous inequality, we obtain

$\sum_{\ell=0}^{p} \varphi_{\beta}^{\ell} \circ\left(i d-\varphi_{\beta}\right)\left(D\left(O_{0, n}(g, s)\right)\right) \leq \sum_{\ell=0}^{p} \varphi_{\beta}^{\ell}\left(d\left(g x_{0}, T x_{0}\right)\right)$.

Using Lemma 1.1, leads to

$\sum_{\ell=0}^{p}\left(i d-\varphi_{\beta}\right)\left(\varphi_{\beta}^{\ell}\left(D\left(O_{0, n}(g, s)\right)\right)\right) \leq \sum_{\ell=0}^{p} \varphi_{\beta}^{\ell}\left(d\left(g x_{0}, T x_{0}\right)\right)$, which implies, by (P4), that

$D\left(O_{0, n}(g, s)\right)-\varphi_{\beta}^{p+1}\left(D\left(O_{0, n}(g, s)\right)\right) \leq \sum_{\ell=0}^{p} \varphi_{\beta}^{\ell}\left(d\left(g x_{0}, T x_{0}\right)\right)$.

Hence, by letting $p \rightarrow \infty$ in the above inequality and using (P2), it follows

$D\left(O_{0, n}(g, s)\right) \leq \sum_{\ell=0}^{\infty} \varphi_{\beta}^{\ell}\left(d\left(g x_{0}, T x_{0}\right)\right), \quad$ for all $\mathrm{n} \in \mathbb{N}$.

Consequently, we have

$D\left(O_{0, \infty}(g, s)\right) \leq \sum_{\ell=0}^{\infty} \varphi_{\beta}^{\ell}\left(d\left(g x_{0}, T x_{0}\right)\right)$.

Lemma 3.4 Under the hypotheses of Lemma 3.2. For all $m, n \in \mathbb{N}$ such that $m>n>0$, we have

$D\left(O_{n, m}(g, s)\right) \leq \varphi_{\beta}^{\ell}\left(D\left(O_{n-\ell, m}(g, s)\right)\right)$ where $1 \leq \ell \leq \mathrm{n}$.

Furthermore, $\left\{g x_{k}\right\}_{k \in \mathbb{N}}$ is a Cauchy sequence.

Proof We proceed by induction on $\ell$. For $\ell=1$, by Lemma 3.2 there exists $n<k \leq m$ such that

$$
\begin{aligned}
D\left(O_{n, m}(g, s)\right)=d\left(g x_{n}, g x_{k}\right) & =d\left(T x_{n-1}, T x_{k-1}\right) \\
& \leq \varphi\left(M_{T, g}\left(x_{n-1}, x_{k-1}\right)\right) \\
& \leq \varphi\left(\beta D\left(O_{n-1, k}(g, s)\right)\right) \\
& =\varphi_{\beta}\left(D\left(O_{n-1, k}(g, s)\right)\right) \\
& \leq \varphi_{\beta}\left(D\left(O_{n-1, m}(g, s)\right)\right) .
\end{aligned}
$$

Assume that the inequality (5) holds for some $\ell<n$. By Lemma 3.2, there exists an integer $k$ with $n-\ell<k \leq m$ such that

$$
\begin{aligned}
D\left(O_{n-\ell, m}(g, s)\right)=d\left(g x_{n-\ell}, g x_{k}\right) & =d\left(T x_{n-\ell-1}, T x_{k-1}\right) \\
& \leq \varphi\left(M_{T, g}\left(x_{n-\ell-1}, x_{k}\right)\right) \\
& \leq \varphi_{\beta}\left(D\left(O_{n-\ell-1, k}(g, s)\right)\right) \\
& \leq \varphi_{\beta}\left(D\left(O_{n-\ell-1, m}(g, s)\right)\right) .
\end{aligned}
$$

Therefore, we obtain

$D\left(O_{n, m}(g, s)\right) \leq \varphi_{\beta}^{\ell}\left(D\left(O_{n-\ell, m}(g, s)\right)\right) \leq \varphi_{\beta}^{\ell+1}\left(D\left(O_{n-\ell-1, m}(g, s)\right)\right)$,

which means inequality (5) holds. Next, we claim that $\left\{g x_{k}\right\}_{k \in \mathbb{N}}$ is a Cauchy sequence. Since we have $d\left(g x_{n}, g x_{m}\right) \leq D\left(O_{n, m}(g, s)\right)$, then for $\ell=n$, we have $d\left(g x_{n}, g x_{m}\right) \leq \varphi_{\beta}^{n}\left(D\left(O_{0, m}(g, s)\right)\right) \leq \varphi_{\beta}^{n}\left(D\left(O_{0, \infty}(g, s)\right)\right)$.

Next, using $\lim _{n \rightarrow+\infty} \varphi_{\beta}^{n}(t)=0$ where $t=D\left(O_{0, \infty}(g, s)\right)$, it follows that for any $\varepsilon>0$, there exists $N>0$ such that 
for all $n \geq N$, we have $\varphi_{\beta}^{n}(t)<\varepsilon$. Since, from Lemma 1.1, we have $\varphi_{\beta}(t)<t$, we conclude that

$d\left(g x_{m}, g x_{n}\right) \leq \varphi_{\beta}^{n}(t) \leq \varphi_{\beta}^{N}(t)<\varepsilon$,

for $m>n>N$. Consequently, $\left\{g x_{k}\right\}_{k \in \mathbb{N}}$ is a Cauchy sequence.

Lemma 3.5 Let $(X, d)$ be a g-orbitally complete space endowed with a binary relation $\mathcal{R}$ such that $(X, d, \mathcal{R})$ is regular. Assume that:

(b1) $T(X) \subset g(X)$;

(b2) There exists $x_{0} \in X$ such that $g x_{0} \mathcal{R} T x_{0}$;

(b3) There exists $\left\{x_{n}\right\}_{n \in \mathbb{N}_{0}} \in \mathcal{O}\left(x_{0}\right)$ such that gx $x_{n}$ converges to $y_{*}=g x_{*}$;

(b4) There exists $\beta>0$ such that $T$ is a $\beta$-quasi contractive mapping;

(b5) $\varphi$ is continuous or $\beta>\max \left\{\alpha_{2}, \alpha_{3}\right\}$.

Then, $x_{*} \in C(g, T)$.

Proof From (b1), (b2) and (b3), we deduce similarly to (4) that for $\left\{x_{n}\right\}_{n \in \mathbb{N}_{0}} \in \mathcal{O}\left(x_{0}\right)$, we have $g x_{n} \mathcal{R} g x_{n+1}$ for all $n \in \mathbb{N}$. By (b3), we have $\lim _{n \rightarrow \infty} d\left(g x_{n}, g x_{*}\right)=0$. Hence, by the regularity of $(X, d, \mathcal{R})$, there exists a subsequence $\left\{g x_{n(k)}\right\}_{k \in \mathbb{N}}$ satisfying $g x_{n(k)} \mathcal{R} g x_{*}, \quad$ for all $\mathrm{k}>0$.

From (b4), we obtain

$d\left(T x_{n(k)}, T x_{*}\right) \leq \varphi\left(M_{T, g}\left(x_{n(k)}, x_{*}\right)\right)$.

Denote $\rho=d\left(g x_{*}, T x_{*}\right)$. For all $k$ we have

$$
\begin{array}{r}
M_{T, g}\left(x_{n(k)}, x_{*}\right)=\max \left\{\alpha_{0} d\left(g x_{n(k)}, g x_{*}\right), \alpha_{1} d\left(g x_{n(k)}, T x_{n(k)}\right),\right. \\
\left.\alpha_{2} d\left(g x_{*}, T x_{*}\right), \alpha_{3} d\left(g x_{n(k)}, T x_{*}\right), \alpha_{4} d\left(g x_{*}, g x_{n(k)+1}\right)\right\} .
\end{array}
$$

Letting $k \rightarrow \infty$ in the above equation, we obtain

$$
\lim _{k \rightarrow \infty} M_{T, g}\left(x_{n(k)}, x_{*}\right)=\max \left\{\alpha_{2} \rho, \alpha_{3} \rho\right\}=\max \left\{\alpha_{2}, \alpha_{3}\right\} \rho .
$$

We claim that $\rho=0$. Suppose, by contradiction, that $\rho>0$. From (b5), if we suppose that $\varphi$ is continuous, we have

$\rho \leq \varphi\left(\max \left\{\alpha_{2}, \alpha_{3}\right\} \rho\right) \leq \varphi(\beta \rho)=\varphi_{\beta}(\rho)<\rho$,

which is a contradiction. However, if we suppose that $\beta>\max \left\{\alpha_{2}, \alpha_{3}\right\}$, then there exists $\varepsilon>0$ and $N>0$ such that for all $n>N$, we have

$$
\begin{aligned}
& M_{T, g}\left(x_{n(k)}, x_{*}\right)<\left(\max \left\{\alpha_{2}, \alpha_{3}\right\}+\varepsilon\right) \rho \text { and } \beta \\
& \quad>\max \left\{\alpha_{2}, \alpha_{3}\right\}+\varepsilon .
\end{aligned}
$$

Therefore,

$$
\begin{aligned}
d\left(g x_{n(k)+1}, T x_{*}\right) & \leq \varphi\left(M_{T, g}\left(x_{n(k)}, x_{*}\right)\right) \\
& \leq \varphi\left(\left(\max \left\{\alpha_{2}, \alpha_{3}\right\}+\varepsilon\right) \rho\right) \\
& =\varphi_{\beta}\left(\frac{\max \left\{\alpha_{2}, \alpha_{3}\right\}+\varepsilon}{\beta} \rho\right) \\
& <\frac{\max \left\{\alpha_{2}, \alpha_{3}\right\}+\varepsilon}{\beta} \rho<\rho .
\end{aligned}
$$

Thus by letting $k \rightarrow \infty$, we get

$\rho<\frac{\max \left\{\alpha_{2}, \alpha_{3}\right\}+\varepsilon}{\beta} \rho<\rho$,

which is a contradiction as well. Hence, our claim holds.

Now, we are ready to furnish the proof of the first main result of the previous section.

Proof of Theorem 2.1 Let $x_{0} \in X$ satisfying (A1) and choose any sequence $s=\left\{x_{n}\right\}_{n \in \mathbb{N}_{0}} \in \mathcal{O}\left(x_{0}\right)$. By Lemma 3.4 , it follows that $\left\{g x_{n}\right\}$ is a Cauchy sequence. As $(X, d)$ is $g$-orbitally complete there exists $y_{*}$ such that $\lim _{n \rightarrow \infty} g x_{n}=y_{*}$. Since $g(X)$ is closed, there exits $x_{*}$ satisfying $y_{*}=g x_{*}$. Suppose that either (A5.a) or (A5.b) holds, then using Lemma 3.5, we deduce that $T x_{*}=g x_{*}$. Now, suppose that (A5.c) holds. Let $s=\left\{x_{n}\right\}_{n \in \mathbb{N}_{0}} \in \mathcal{O}\left(x_{0}\right)$ such that $T$ and $g$ commute at the orbit $O_{\infty}(g, s)$. Since the sequence $\left\{g x_{n}\right\}$ is a Cauchy sequence, let $y_{*}=\lim _{n \rightarrow \infty} g x_{n}$. Since $T$ and $g$ commute at the orbit, for all $n \geq 2$, we have $g^{2} x_{n}=T^{2} x_{n-2}$, then $\lim _{n \rightarrow \infty} g^{2} x_{n}=\lim _{n \rightarrow \infty} T^{2} x_{n-2}$. Using the continuity of $T$ and $g$, we get

$$
g\left(\lim _{n \rightarrow \infty} g x_{n}\right)=T\left(\lim _{n \rightarrow \infty} T x_{n-2}\right),
$$

which implies $g y_{*}=T y_{*}$.

In order to prove Theorem 2.2, we will first need the following two lemmas:

Lemma 3.6 In addition to the hypotheses of Theorem 2.2, suppose that $x_{*}, y_{*} \in C(g, T)$, then $g x_{*}=g y_{*}$.

Proof As $C(g, T)$ is $\mathcal{R}$-g-directed, there exists $x_{0} \in X$ such that $g x_{*} \mathcal{R} g x_{0}$ and $g y_{*} \mathcal{R} g x_{0}$. We consider a sequence $s=\left\{x_{n}\right\}_{n \in \mathbb{N}_{0}} \in \mathcal{O}\left(x_{0}\right)$. First, lets assume that $\mathcal{R}$ is symmetric, then $g x_{0} \mathcal{R} T x_{0}$. Hence, by Lemma 3.4, the sequence $\left\{g x_{n}\right\}$ is a Cauchy sequence. Since $(X, d)$ is $g$-orbitally complete, there exists $z_{*}$ such that $\lim _{n \rightarrow+\infty} g x_{n}=z_{*}$. As the relation $\mathcal{R}$ is transitive we have $g x_{n} \mathcal{R} g x_{*}$. Using the fact that $T$ is $\beta$-quasi contractive we obtain

$$
\begin{aligned}
& d\left(g x_{n+1}, g x_{*}\right)=d\left(T x_{n}, T x_{*}\right) \\
& \leq \varphi\left(\operatorname { m a x } \left\{\alpha_{0} d\left(g x_{n}, g x_{*}\right), \alpha_{1} d\left(g x_{n}, g x_{n+1}\right), \alpha_{3} d\left(g x_{n}, g x_{*}\right),\right.\right. \\
& \left.\left.\quad \alpha_{4} d\left(g x_{*}, x_{n}\right)\right\}\right) \leq \varphi_{\beta}\left(\max \left\{d\left(g x_{*}, g x_{n}\right), d\left(g x_{n}, g x_{n+1}\right)\right\}\right) .
\end{aligned}
$$


If there exists a subsequence $\{n(k)\}_{k \in \mathbb{N}_{0}}$ such that

$d\left(g x_{n(k)}, g x_{n(k)+1}\right)>d\left(g x_{*}, g x_{n(k)}\right)$,

then, we have

$d\left(g x_{n(k)+1}, g x_{*}\right) \leq \varphi_{\beta}\left(d\left(g x_{n(k)}, g x_{n(k)+1}\right)\right)<d\left(g x_{n(k)}, g x_{n(k)+1}\right)$,

and therefore $\lim _{k \rightarrow \infty} d\left(g x_{n(k)+1}, g x_{*}\right)=0$ and hence $z_{*}=g x_{*}$. If there is no subsequence $n(k)$ such that (6) is true, then there exists $N>0$ such that for all $n>N$, we haved $\left(g x_{n}, g x_{*}\right)>d\left(g x_{n}, g x_{n+1}\right)$, and it follows

$d\left(g x_{n+1}, g x_{*}\right) \leq \varphi_{\beta}\left(d\left(g x_{n}, g x_{*}\right)\right) \leq \varphi_{\beta}^{n-N}\left(d\left(g x_{N}, g x_{*}\right)\right)$,

which converges to 0 as $n$ tends to infinity. Hence, $\lim _{n \rightarrow \infty} d\left(g x_{n(k)}, g x_{*}\right)=0$ and then $z_{*}=g y_{*}$. Similarly, we prove that $z_{*}=g y_{*}$ which implies that $g x_{*}=g y_{*}$.

Now, assume that $\beta \geq \max _{0 \leq k \leq 4}\left\{\alpha_{k}, 2 \alpha_{1}\right\}$. As $T$ is a $g$-comparative mapping, for all $n \geq 0$, we have $g x_{*} \mathcal{R} g x_{n}$ and $g y_{*} \mathcal{R} g x_{n}$. Since $T$ is $\beta$-quasi contractive, we have

$d\left(g x_{n+1}, g x_{*}\right)=d\left(T x_{n}, T x_{*}\right) \leq \varphi\left(M_{T, g}\left(x_{n}, x_{*}\right)\right)$.

On the other hand, we have

$$
\begin{aligned}
M_{T, g}\left(x_{n}, x_{*}\right)= & \max \left\{\alpha_{0} d\left(g x_{n}, g x_{*}\right), \alpha_{1} d\left(g x_{n}, g x_{n+1}\right),\right. \\
& \left.\alpha_{3} d\left(g x_{n}, g x_{*}\right), \alpha_{4} d\left(g x_{*}, g x_{n+1}\right)\right\} \\
\leq & \max \left\{\alpha_{0} d\left(g x_{n}, g x_{*}\right), \alpha_{1} d\left(g x_{n}, g x_{*}\right)\right. \\
& +\alpha_{1} d\left(g x_{*}, g x_{n+1}\right), \\
& \left.\alpha_{3} d\left(g x_{n}, g x_{*}\right), \alpha_{4} d\left(g x_{*}, g x_{n+1}\right)\right\} \\
\leq & \beta \max \left\{d\left(g x_{n}, g x_{*}\right), d\left(g x_{n+1}, g x_{*}\right)\right\} .
\end{aligned}
$$

And as $\varphi$ is nondecreasing, we have

$$
\begin{gathered}
d\left(g x_{n+1}, g x_{*}\right)=d\left(T x_{n}, T x_{*}\right) \leq \varphi\left(M_{T, g}\left(x_{n}, x_{*}\right)\right) \\
\leq \varphi_{\beta}\left(\max \left\{d\left(g x_{n}, g x_{*}\right), d\left(g x_{n+1}, g x_{*}\right)\right\}\right) .
\end{gathered}
$$

Suppose that for some $n$ we have $d\left(g x_{n}, g x_{*}\right) \leq$ $d\left(g x_{n+1}, g x_{*}\right)$. It follows that

$d\left(g x_{n+1}, g x_{*}\right) \leq \varphi_{\beta}\left(d\left(g x_{n+1}, g x_{*}\right)\right)<d\left(g x_{n+1}, g x_{*}\right)$,

which is a contradiction.

Then for all $n \geq 0$, we necessary have $d\left(g x_{n}, g x_{*}\right)$ $>d\left(g x_{n+1}, g x_{*}\right)$, and it follows that

$d\left(g x_{n+1}, g x_{*}\right) \leq \varphi_{\beta}\left(d\left(g x_{n}, g x_{*}\right)\right)$.

Then, by induction we obtain that

$d\left(g x_{n+1}, g x_{*}\right) \leq \varphi_{\beta}^{n}\left(d\left(g x_{0}, g x_{*}\right)\right)$,

which implies that $\lim _{n \rightarrow \infty} d\left(g x_{n}, g x_{*}\right)=0$. Similarly, we can prove that

$\lim _{n \rightarrow \infty} d\left(g x_{n}, g y_{*}\right)=0$.

Consequently, we have $g x_{*}=g y_{*}$.
Lemma 3.7 Let $X$ be a non empty set and $T, g: X \rightarrow X$ be two mappings such that:

(c1) $\quad C(g, T)$ is non empty.

(c2) $T$ and $g$ commute at any coincidence point.

(c3) $g x_{*}=g y_{*}$ for all $x_{*}, y_{*}$ in $C(g, T)$.

Then $T$ and $g$ have a unique common fixed point.

Proof Let $x_{*} \in C(g, T)$, that is, $g x_{*}=T x_{*}$. Since $T$ and $g$ commute at their coincidence points, then

$g^{2} x_{*}=g T x_{*}=T g x_{*}$.

Let us denote $z=g x_{*}$. Then from the above equation, it follows that $g z=T z$. Thus, $z$ is a coincidence point. By (c3), we have $g z=g x_{*}=z=T z$, that is, $z$ is a common fixed point of $T$ and $g$. This common fixed point is unique; assuming $\omega$ is another common fixed point of $T$ and $g$, then again by (c3) we have $\omega=g \omega=g z=z$.

Proof of Theorem 2.2 By Theorem 2.1 $C(g, T)$ is non empty. Then by Lemma 3.6, the hypotheses of Lemma 3.7 are satisfied. Therefore $T$ and $g$ have a unique common fixed point.

Proof of Theorem 2.3 Let $s=\left\{x_{n}\right\}_{n \in \mathbb{N}_{0}} \in \mathcal{O}\left(x_{0}\right)$. By Lemma 3.1 we have

$d\left(g x_{n+1}, g x_{n}\right)=d\left(T x_{n}, T x_{n-1}\right) \leq \varphi\left(M_{T, g}\left(x_{n}, x_{n-1}\right)\right)$.

On the other hand, for all $n \geq 1$, we have:

$$
\begin{aligned}
M_{T, g}\left(x_{n}, x_{n-1}\right)= & \max \left\{\alpha_{0} d\left(g x_{n}, g x_{n-1}\right), \alpha_{1} d\left(g x_{n}, T x_{n}\right),\right. \\
& \left.\alpha_{2} d\left(g x_{n-1}, T x_{n-1}\right), \alpha_{3} d\left(g x_{n}, T x_{n-1}\right), \alpha_{4} d\left(g x_{n-1}, T x_{n}\right)\right\} \\
= & \max \left\{\alpha_{0} d\left(g x_{n}, g x_{n-1}\right), \alpha_{1} d\left(g x_{n}, g x_{n+1}\right),\right. \\
& \left.\alpha_{2} d\left(g x_{n-1}, g x_{n}\right), \alpha_{4} d\left(g x_{n-1}, g x_{n+1}\right)\right\} \\
\leq & \max \left\{\alpha_{0} d\left(g x_{n-1}, g x_{n}\right), \alpha_{1} d\left(g x_{n}, g x_{n+1}\right),\right. \\
& \left.\alpha_{2} d\left(g x_{n}, g x_{n-1}\right), \alpha_{4} d\left(g x_{n-1}, g x_{n}\right)+\alpha_{4} d\left(g x_{n}, g x_{n+1}\right)\right\} \\
\leq & \beta \max \left\{d\left(g x_{n-1}, g x_{n}\right), d\left(g x_{n}, g x_{n+1}\right)\right\} .
\end{aligned}
$$

Since $\varphi$ is nondecreasing, we have

$\varphi\left(M_{T, g}\left(x_{n}, x_{n-1}\right)\right) \leq \varphi\left(\beta \max \left\{d\left(g x_{n-1}, g x_{n}\right), d\left(g x_{n}, g x_{n+1}\right)\right\}\right)$.

Assume that $d\left(g x_{n}, g x_{n-1}\right) \leq d\left(g x_{n}, g x_{n+1}\right)$. From (7), (8) and Lemma 1.1, we obtain

$d\left(g x_{n+1}, g x_{n}\right) \leq \varphi_{\beta}\left(d\left(g x_{n}, g x_{n+1}\right)\right)<d\left(g x_{n}, g x_{n+1}\right)$,

which is a contradiction.

Then, for all $n$ we have $d\left(g x_{n}, g x_{n-1}\right)>d\left(g x_{n}, g x_{n+1}\right)$. By (8) we have

$$
\begin{aligned}
d\left(g x_{n}, g x_{n+1}\right) & \leq \varphi\left(\beta d\left(g x_{n}, g x_{n-1}\right)\right)=\varphi_{\beta}\left(d\left(g x_{n}, g x_{n-1}\right)\right) \\
& \leq \varphi_{\beta}^{2}\left(d\left(g x_{n-1}, g x_{n-2}\right)\right) \leq \cdots \leq \varphi_{\beta}^{n}\left(d\left(g x_{1}, g x_{0}\right)\right) .
\end{aligned}
$$

In addition, for $n<m$ we have 


$$
\left.d\left(g x_{n}, g x_{m}\right) \leq \sum_{k=n}^{m-1} d\left(g x_{k}, g x_{k+1}\right)\right) \leq \sum_{k=n}^{m-1} \varphi_{\beta}^{k}\left(d\left(g x_{1}, g x_{0}\right)\right) .
$$

Since, from (P2), we have $\sum_{n=1}^{\infty} \varphi_{\beta}^{n}(t)<\infty$, then for every $\varepsilon>0$ there exists $N>0$ such that

$\sum_{k=n}^{m-1} \varphi_{\beta}^{k}(t)<\varepsilon \quad$ forallm $>\mathrm{n}>\mathrm{N}$.

Thus $d\left(g x_{n}, g x_{m}\right)<\varepsilon$. This implies that $\left\{g x_{n}\right\}$ is a Cauchy sequence in $X$. Now, since $(X, d)$ is complete and $g(X)$ is closed, then there exists $x_{*} \in X$ such that $g x_{*}=\lim _{n \rightarrow \infty} g x_{n}$. And finally, using Lemma 3.5 we conclude that $g x^{*}=T x^{*}$. That is $x^{*}$ is a coincidence point.

Proof of Theorem 2.4 Since $C(g, T)$ is $\mathcal{R}$ - $g$-directed, then there exists $x_{0} \in X$ such that $g x_{*} \mathcal{R} g x_{0}$ and $g y_{*} \mathcal{R} g x_{0}$. Let $s=\left\{x_{n}\right\}_{n \in \mathbb{N}_{0}} \in \mathcal{O}\left(x_{0}\right)$, as $T$ is a $g$-comparative mapping, for all $n \geq 0$, we have $g x_{*} \mathcal{R} g x_{n}$ and $g y_{*} \mathcal{R} g x_{n}$ and then

$d\left(g x_{n+1}, g x_{*}\right)=d\left(T x_{n}, T x^{*}\right) \leq \varphi\left(M_{T, g}\left(x_{n}, x_{*}\right)\right)$.

On the other hand, we have

$$
\begin{aligned}
M_{T, g}\left(x_{n}, x_{*}\right)= & \max \left\{\alpha_{0} d\left(g x_{n}, g x_{*}\right), \alpha_{1} d\left(g x_{n}, g x_{n+1}\right), \alpha_{3} d\left(g x_{n}, g x_{*}\right),\right. \\
& \left.\alpha_{4} d\left(g x_{*}, g x_{n+1}\right)\right\} \\
\leq & \max \left\{\alpha_{0} d\left(g x_{n}, g x_{*}\right), \alpha_{1} d\left(g x_{n}, g x_{*}\right)+\alpha_{1} d\left(g x_{*}, g x_{n+1}\right),\right. \\
& \left.\alpha_{3} d\left(g x_{n}, g x_{*}\right)+\alpha_{4} d\left(g x_{*}, g x_{n+1}\right)\right\} \\
\leq & \beta \max \left\{d\left(g x_{n}, g x_{*}\right), d\left(g x_{n+1}, g x_{*}\right)\right\} .
\end{aligned}
$$

As a consequence, using (9), we have

$$
d\left(g x_{n+1}, g x_{*}\right) \leq \varphi_{\beta}\left(\max \left\{d\left(g x_{n}, g x_{*}\right), d\left(g x_{n+1}, g x_{*}\right)\right\}\right) .
$$

Next, we shall prove that

$\lim _{n \rightarrow \infty} d\left(g x_{n}, g x_{*}\right)=0$.

Suppose for some $n$, we have $d\left(g x_{n}, g x_{*}\right) \leq d\left(g x_{n+1}, g x_{*}\right)$. We have from (10)

$d\left(g x_{n+1}, g x_{*}\right) \leq \varphi_{\beta}\left(d\left(g x_{n+1}, g x_{*}\right)\right)<d\left(g x_{n+1}, g x_{*}\right)$,

which is a contradiction.

Then for all $n \geq 0$, we have $d\left(g x_{n}, g x_{*}\right)>d\left(g x_{n+1}, g x_{*}\right)$. And, using (10), we have $d\left(g x_{n+1}, g x_{*}\right) \leq \varphi_{\beta}\left(d\left(g x_{n}, g x_{*}\right)\right)$ for all $n$. By induction we obtain

$d\left(g x_{n+1}, g x_{*}\right) \leq \varphi_{\beta}^{n}\left(d\left(g x_{0}, g x_{*}\right)\right)$ forall n.

Hence (11) follows from (P2). Similarly, we can prove that $\lim _{n \rightarrow \infty} d\left(g x_{n}, g y_{*}\right)=0$.

Therefore, by the uniqueness of the limit, it follows that $g x_{*}=g y_{*}$ and by Lemma 3.7, $T$ and $g$ have a unique common fixed point.

\section{Consequences}

Several consequences of the main results are established in this section.

Corollary 4.1 ([3]) Let $(X, d)$ be a complete space endowed with a transitive binary relation $\mathcal{R}$ such that $(X, d, \mathcal{R})$ is regular. Let $T: X \rightarrow X$ be a mapping satisfying the following conditions:

(A1) there exists $x_{0} \in X$ such that $x_{0} \mathcal{R} T x_{0}$;

(A2) $T$ is id-comparative;

(A3) there exists $\varphi \in \Phi_{2}$ such that

$$
\begin{aligned}
& d(T x, T y) \leq \varphi\left(\operatorname { m a x } \left\{d(x, y), \frac{d(x, T x)+d(y, T y)}{2},\right.\right. \\
& \left.\left.\frac{d(x, T y)+d(y, T x)}{2}\right\}\right)
\end{aligned}
$$

Then, T has a a fixed point $x_{*} \in X$. Moreover, if in addition, $C(i d, T)$ is $\mathcal{R}$-id-directed, then $x_{*}$ is the unique fixed point of $T$ in $X$.

Proof This result follows immediately from Theorem 2.1 and Theorem 2.2 by taking $g=i d$.

Remark 4.1 Noting that Samet and Turinici in [3] proved the previous result for $\varphi \in \Phi_{1}$ and without condition (P4). However, the existence of the fixed point can be proved for a transitive binary relation but not necessarily symmetric.

Corollary 4.2 Let $(X, d)$ a complete metric space endowed with a partial order $\leq$ such that $(X, d, \leq)$ is regular. Let $g, T: X \rightarrow X$ two mappings satisfying the following conditions:

(1) there exists $x_{0} \in X$ such that $g x_{0} \leq T x_{0}$;

(2) $g(X)$ is closed and $T(X) \subset g(X)$;

(3) $g x \leq$ gy implies $T x \leq T y$ for all $x, y \in X$;

(4) there exists $\beta>0$ and a continuous function $\varphi \in \Phi_{\beta}$ such that

$x, y \in X: g x \leq g y \Longrightarrow d(T x, T y) \leq \varphi\left(M_{g, T}(x, y)\right)$,

where $M_{g, T}(x, y)$ is given by Definition 1.6.

Then, $T$ and $g$ have a coincidence point in $X$.

Proof The coincidence point result follows immediately from Theorem 2.1 by taking a binary relation $\mathcal{R}$ given by $x, y \in X: x \mathcal{R} y \Longleftrightarrow x \leq y$.

Corollary 4.3 Let $(X, d)$ be a complete metric space endowed with a partial order $\leq$ such that $(X, d, \leq)$ is regular. Let $T: X \rightarrow X$ be a mapping satisfying the following conditions: 
(1) There exists $x_{0} \in X$ such that $x_{0} \leq T x_{0}$;

(2) $T$ is non decreasing;

(3) There exists a continuous function $\varphi \in \Phi_{1}$ such that

$$
\begin{aligned}
& d(T x, T y) \leq \varphi(\max \{d(x, y), d(x, T x), d(y, T y), \\
& d(x, T y), d(y, T x)\}),
\end{aligned}
$$

for all $x \leq y$. Then, $T$ has a fixed point in $X$.

Proof The fixed point result comes as an immediate consequence of Corollary 4.2 when $g=i d$.

Corollary 4.4 Let $(X, d)$ be a complete metric space, $T, g: X \rightarrow X$ be a pair of commuting continuous mappings, such that $g(X)$ is closed and $T(X) \subset g(X)$. Assume that there exists $\varphi \in \Phi_{1}$ satisfying

$$
\begin{aligned}
& d(T x, T y) \leq \varphi(\max \{d(g x, g y), d(g x, T x), d(g y, T y), \\
& d(g x, T y), d(g y, T x)\}),
\end{aligned}
$$

for all $x, y \in X$. Then, $T$ and $g$ have a unique common fixed point.

Proof All hypotheses of Theorem 2.1 are satisfied by taking $\mathcal{R}=X \times X$. In addition hypothesis (A5.c) is also satistied, and therefore we have shown the existence of a coincidence point. Furthermore, all hypotheses of Theorem 2.2 are satisfied, which implies that $T$ and $g$ have a unique common fixed point.

Corollary 4.5 Let $(X, d)$ be a complete metric space and $T, g: X \rightarrow X$ be two commuting mappings such that $g(X)$ is closed and $T(X) \subset g(X)$. Suppose there exists $q \in(0,1)$ satisfying

$$
\begin{aligned}
& d(T x, T y) \leq q \max \{d(g x, g y), d(g x, T x), d(g y, T y), \\
& \quad d(g x, T y), d(g y, T x)\},
\end{aligned}
$$

for all $x, y \in X$. Then, $T$ and $g$ have a unique common fixed point.

Proof It is an immediate consequence of the previous corollary where $\varphi(t)=q t$.

Corollary 4.6 ([16]) Let $(X, d)$ be a complete metric space and $T: X \rightarrow X$ be continuous mapping. Suppose there exists $\varphi \in \Phi_{1}$ such that

$d(T x, T y) \leq \varphi(\max \{d(x, y), d(x, T x), d(y, T y), d(x, T y), d(y, T x)\})$,

for all $x, y \in X$. Then, $T$ has a unique fixed point in $X$.

Proof The result is an immediate consequence of Corollary 4.4 when $g=I d$.

Remark 4.2 The condition (P4) from Definition 1.5 is not introduced in [16]. However, it is required to prove [Lemma 2, [16]].
Corollary 4.7 ([2]) Let $(X, d)$ be a complete metric space and $T: X \rightarrow X$ be continuous mapping. Suppose that (1) holds. Then, $T$ has a unique fixed point in $X$.

Proof This result is an immediate consequence of Corollary 4.5 with $g=i d$.

Remark 4.3 Further consequences may be derived once we specify the binary relation $\mathcal{R}$.

\section{Applications}

Integral equations govern many topics in several disciplines, such as applied mechanics, population dynamics, and economy. They arise from several applications (see, for instance, Agarwal et al. [20] and O'Regan and Meehan [21] and the references therein). As an example, we will apply Theorem 2.1 to establish some conditions which guarantee the existence of solutions for some generalized Urysohn integral equation

$$
g x(t)=\int_{0}^{t} F(t, s, x(s)) d s+\lambda(t), t \in[0, \tau],
$$

where $F:[0, \tau] \times[0, \tau] \times \mathbb{R}^{n} \rightarrow \mathbb{R}^{n}, \quad \lambda:[0, \tau] \rightarrow \mathbb{R}^{n}$ are continuous and $g: X \rightarrow X$ surjective.

Let $\left(X,\|\cdot\|_{B}\right)$ be a Banach space. The space $X=$ $C([0, \tau])$ is endowed with the Bielecki-type norm which is given by

$$
\|x\|_{\kappa, \tau}=\max _{t \in[0, \tau]} e^{-\kappa t}|x(t)|, \quad \text { for all } \mathrm{x} \in \mathrm{X}
$$

where $\kappa>0$, is arbitrarily chosen. Define

$$
d_{\kappa, \tau}(x, y)=\|x-y\|_{\kappa, \tau}=\max _{t \in[0, \tau]} e^{-\kappa t}|x(t)-y(t)|, \text { for all, } \mathrm{x}, \mathrm{y} \in \mathrm{X} \text {. }
$$

Noting that $\left(X, d_{\kappa, \tau}\right)$ is a complete metric space.

Let $\xi: \mathbb{R}^{n} \times \mathbb{R}^{n} \rightarrow \mathbb{R}$ be a function and $\tau \geq 1$. Assume that the following assumption holds:

(H1) for all $u, v, w \in \mathbb{R}^{n}$, if $\xi(u, v) \geq 0$ and $\xi(v, w) \geq 0$, then $\xi(u, w) \geq 0$;

(H2) for all $x, y \in X$ such that $\xi(g x(s), g v(s)) \geq 0$ for all $s \in[0, \tau]$ we have

$$
\begin{aligned}
& |F(t, s, x(s))-F(t, s, y(s))| \leq \varphi(2|g x(s)-g y(s)|), \\
& \quad \text { for all s } \in[0, \tau],
\end{aligned}
$$

where $\varphi \in \Phi_{2}$ and $\varphi(\alpha t) \leq \alpha \varphi(t)$ for all $t \in[0, \tau]$ and for all $\alpha \geq 1$.

(H3) there exists $x_{0} \in X$ such that for all $t \in[0, \tau]$,

$$
\xi\left(g x_{0}(t), \int_{0}^{t} F\left(t, s, x_{0}(s)\right) d s+\lambda(t)\right) \geq 0 \text {. }
$$


(H4) for all $x, y \in X$ and for all $t \in[0, \tau]$, if $\xi(g x(t), g y(t)) \geq 0$, then

$$
\xi\left(\int_{0}^{t} F(t, s, x(s)) d s+\lambda(t), \int_{0}^{t} F(t, s, y(s)) d s+\lambda(t)\right) \geq 0 .
$$

(H5) if $\left\{x_{n}\right\}$ is a sequence in $X$ such that $\lim _{n \rightarrow \infty} d_{\kappa, \tau}\left(x_{n}, x\right)=0$ and $\xi\left(x_{n}(t), x_{n+1}(t)\right) \geq 0$ for all $n \in \mathbb{N}_{0}$ and $t \in[0, \tau]$ then $\xi\left(x_{n}(t), x(t)\right) \geq 0$ for all $n \in \mathbb{N}_{0}$ and for all $t \in[0, \tau]$.

Theorem 5.1 Under hypotheses (H1)-(H5), the integral equation (12) has at least one solution $x_{*}$ in $X$.

Proof Define $T$ by

$T x(t)=\int_{0}^{t} F(t, s, x(s)) d s+\lambda(t), \quad t \in[0, \tau]$.

Let $x, y \in X$ satisfying $\xi(g x(s), g y(s)) \geq 0$ for all $t \in[0, \tau]$. Therefore, from $(\mathrm{H} 2)$, we have

$$
\begin{aligned}
|T x(t)-T y(t)| & =\left|\int_{0}^{t} F(t, s, x(s))-F(t, s, y(s)) d s\right| \\
& \leq \int_{0}^{t}|F(t, s, x(s))-F(t, s, y(s))| d s \\
& \leq \int_{0}^{t} \varphi(2|g x(s)-g y(s)|) d s \\
& \leq \int_{0}^{t} \varphi\left(2|g x(s)-g y(s)| e^{-\tau s} e^{\tau s}\right) d s \\
& \leq \varphi\left(2 d_{\kappa, \tau}(g x, g y)\right) \int_{0}^{t} e^{\tau s} d s \\
& \leq \varphi\left(2 d_{\kappa, \tau}(g x, g y)\right) \int_{0}^{t} e^{\tau s} d s \\
& \leq \tau^{-1} \varphi\left(2 d_{\kappa, \tau}(g x, g y)\right) e^{\tau t} .
\end{aligned}
$$

Therefore, for $\tau \geq 1$, we obtain

$d_{\kappa, \tau}(T x, T y) \leq \varphi\left(2 d_{\kappa, \tau}(g x, g y)\right)$.

Next, we define the binary relation by

$x \mathcal{R} y \quad$ if and only if $\quad \xi(\mathrm{x}(\mathrm{t}), \mathrm{y}(\mathrm{t})) \geq 0$, for all $\mathrm{t} \in[0, \tau]$.

Then, we have

$$
g x \mathcal{R} g y \Longrightarrow d_{\kappa, \tau}(T x, T y) \leq \varphi\left(2 d_{\kappa, \tau}(g x, g y)\right) \text {. }
$$

Hence, by Theorem 2.1 for $M_{T, g}=2 d(g x g y)$, it follows that $T$ and $g$ have at least a coincidence point in $X$, that is, $T x_{*}=g x_{*}$. Consequently, the integral equation (12) has at least one solution $x_{*}$ in $X$.

\section{Conclusion}

Improvements to some coincidence and common fixed point theorems are proposed. In particular, the result due to Ćirić in [2] for quasi-contractive mapping is generalized. Furthermore, the fixed point theorem due to Samet and Turinici in [3] for spaces endowed with binary relation has been extended. This has been achieved by introducing the $\beta$-quasi contractive mappings on metric spaces endowed with binary relations involving $\beta$-comparison functions. As an application, the existence of solutions to a generalized class of Urysohn integral equations has been shown. The approach used in the current contribution may be extended for different types of metric spaces.

Open Access This article is distributed under the terms of the Creative Commons Attribution 4.0 International License (http://crea tivecommons.org/licenses/by/4.0/), which permits unrestricted use, distribution, and reproduction in any medium, provided you give appropriate credit to the original author(s) and the source, provide a link to the Creative Commons license, and indicate if changes were made.

\section{References}

1. Banach, S.: Sur les opérations dans les ensembles abstraits et leur application aux équations intégrales. Fund. Math. 3(1), 133-181 (1922)

2. Ćirić, L.B.: A generalization of Banach's contraction principle. Proc. Am. Math. Soc. 45(2), 267-273 (1974)

3. Samet, B., Turinci, M.: Fixed points theorems on a metric space endowed with an arbitrary binary relation and applications. Commun. Math. Anal. 13, 82-97 (2012)

4. Kannan, R.: On certain sets and fixed point theorems. Roum. Math. Pure. Appl. 14, 51-54 (1969)

5. Chatterjea, S. K.: Fixed-point theorems. C.R. Acad. Bulgare Sci. 25, pp 727-730 (1972)

6. Hardy, G.E., Rogers, T.D.: A generalization of a fixed point theorem of Reich. Canad. Math. Bull. 16, 201-206 (1973)

7. Ran, A.C.M., Reurings, M.C.B.: A fixed point theorem in partially ordered sets and some applications to matrix equations. Proc. Am. Math. Soc. 132, 1435-1443 (2003)

8. Nieto, J. J., López, R. R.: Existence and uniqueness of fixed point in partially ordered sets and applications to ordinary differential equations. Acta Math. Sin. (Engl. Ser.) 23, 2205-2212 (2007)

9. Ćirić, L.B.: Generalized contractions and fixed point theorems. Publ. Inst. Math. 12, 19-26 (1971)

10. Kirk, W.A., Srinivasan, P.S., Veeramani, P.: Fixed points for mappings satisfying cyclical contractive conditions. Fixed Point Theory. 4(1), 79-89 (2003)

11. Berzig, M.: Coincidence and common fixed point results on metric spaces endowed with an arbitrary binary relation and applications. J. Fixed Point Theory Appl. 12(1-2), 221-238 (2012) 
12. Shahzad, N., Karapınar, E., Roldán, A.: On some fixed point theorems under $(\alpha, \psi, \phi)$-contractivity conditions in metric spaces endowed with transitive binary relations. Fixed Point Theory Appl. 2015(104), 1-24 (2015)

13. Berzig, M., Karapınar, E., Roldán, A.: Discussion on generalized$(\alpha \psi, \beta \varphi)$-contractive mappings via generalized altering distance function and related fixed point theorems, Abstract and Applied Analysis, Article Id: 259768 (2013)

14. Berzig, M., Karapınar, E.: Fixed point results for $(\alpha \psi, \beta \varphi)$-contractive mappings for a generalized altering distance. Fixed Point Theory Appl. 1, 1-18 (2013)

15. Berzig, M., Rus, M.-D.: Fixed point theorems for $\alpha$-contractive mappings of Meir-Keeler type and applications. Nonlinear Anal. Model. Control 19(2), 178-198 (2014)

16. Khan, M.S., Berzig, M., Chandok, S.: Fixed point theorems in bimetric space endowed with binary relation And Applications. Miskolc Math. Notes 16(2), 939-951 (2015)
17. Aydi, H., Berzig, M.: Coincidence point theorems in higher dimension for nonlinear contractions. J. Nonlinear Anal. Optim 4(1), 53-64 (2012)

18. Alam, A., Imdad, M.: Nonlinear contractions in metric spaces under locally $T$-transitive binary relations. arXiv: 1512.00348 (2016)

19. Turinici, M.: Contractive operators in relational metric spaces. In handbook of functional equations. Springer, New York (2014)

20. Agarwal, R.P., O'Regan, D., Wong, P.J.: Positive solutions of differential. Difference and integral equations. Dordrecht, The Netherlands (1999)

21. O'Regan, D., Meehan, M.M.: Existence Theory for nonlinear integral and integrodifferential equations. Kluwer Academic Publishers, Dordrecht (1998) 